\title{
Sensitivity Analysis for Coupled Structural-Acoustic System with Absorbing Material Using FEM/BEM
}

\author{
Xiuyun Chen(1), Ling Yao(2), Ruhui Cheng(2), Xiaohui Yuan(2), Yanming $X \mathbf{u}^{\left(1,3^{*}\right)}$ \\ (1) School of Architectural Engineering, Huanghuai University, Zhumadian 463003, Henan, P.R. CHINA \\ e-mail: chenxiuyun2004@163.com \\ (2) College of Architecture and Civil Engineering, Xinyang Normal University, Xinyang, 464000, CHINA \\ (3) CAS Key Laboratory of Mechanical Behavior and Design of Materials, Department of Modern Mechanics, University of \\ Science and Technology of China, Hefei, 230026, CHINA \\ (*) the Corresponding Author: cat2@mail.ustc.edu.cn
}

\section{SUMMARY}

Since the acoustic impedance in water cannot be neglected with respect to the mechanical impedance, the acoustic radiation caused by the vibration of structures in the compressible fluid would react to the structure. Therefore, the fluid-structure interaction needs to be considered. The finite element method is used for structure vibration analysis and the boundary element method for acoustic analysis. Sound absorption materials are used to reduce the scattering sound field in the reference region. The sensitivity analysis of a fully coupled structural-acoustic system is proposed. Numerical tests verify the correctness of the proposed algorithm.

KEY WORDS: Boundary element method; Finite element method; Sensitivity analysis; Absorbing material.

\section{INTRODUCTION}

The acoustic radiation and scattering of elastomers immersed in water is a very important research topic to which the engineering field has paid great attention. For example, the underwater radiated noise from ships or submarines affects their concealment and reduces the naval and hydroacoustic equipment performance. In the vibration and noise prediction of large and complex structures such as ships or submarines, the effect of water on the vibration of structures cannot be ignored because the mechanical impedance of structures is only several times larger than the acoustic impedance of water. Many researchers have addressed this problem. Some argue that analytical method, such as the separation of variables, can be applied for simple structures such as spherical shells or infinite cylindrical shells under axisymmetric loads. Another classic analytical method to analyse the acoustic structure coupling of cylindrical shell or spherical shell under the action of plane waves is the resonance scattering theory. Although the analytical methods have played an important role in solving 
structure-acoustic coupling problems, it is usually difficult to predict the response of complex structures. Therefore, it is necessary to develop appropriate numerical methods to solve the acoustic-vibration coupling problems.

At present, the finite element method (FEM) is undoubtedly the most widely used and the most mature development in the field of structural vibration analysis. The boundary element method (BEM) is favored for its unique advantages in the analysis of external acoustic field problems. Therefore, the combination of the FEM and the BEM effectively solves the acousticvibration coupling problem. This method was used for the first time by Everstine and Henderson [1] and attracted the attention of many scholars [2-7]. The vibration and acoustic analysis need to consider the interaction between sound radiation and structural vibration. The difference in radiation medium is directly related to the feedback effect of acoustic radiation on structural vibration since the sound radiation is greatly affected by the medium. In low impedance media such as air, the acoustic radiation has little effect on the structure vibration [8] and is called weak coupling analysis. In the weak coupling analysis, effects of structure vibration are numerically investigated using the finite element method (FEM) first. The finite element solutions of structure vibratory velocity are then used as the boundary condition for consequent acoustic radiation calculation using the boundary element method (BEM) [9]. The same procedure can be used to analyse the rock-socketed pile in isotropic saturated media [10-11].

For the high-density fluid, the effect of structure on the acoustic field and the feedback effect of acoustic radiation on the structure should be considered in coupling analysis [12-13]. This situation is called strong coupling analysis. Fischer and Gaul [14] propose an algorithm by coupling finite elements to a Galerkin boundary element method. Peters and Marburg demonstrate the feasibility of calculating the coupling matrix for interfacing elements with quadratic shape functions [15]. To complete the procedure of FEM-BEM coupling, an iterative process is applied to the common boundary where the variables will converge towards the constant value. This method is put forward to simulate acoustic-elastic waves through axisymmetric media [16]. The fast multipole method is applied to increase the efficiency of acoustic radiation analysis [17-21]. The use of fast multipole algorithm in acoustics is divided into the low-frequency algorithm and high-frequency algorithm, according to the range of analysis frequency. To improve the efficiency and stability of the proposed algorithm, a broadband fast multipole algorithm is proposed for combining the two methods [22-27]. In the paper, we consider the effect of sound-absorbing materials on the coupling system. A coupled system equation with the impedance characteristics of sound-absorbing materials is established and the sensitivity formulation is presented.

This paper is organized as follows: Section 2 introduces vibro-acoustic analysis by coupling boundary element formulation and finite element formulation, Section 3 discusses the main steps of sensitivity analysis and Section 4 provides numerical examples to prove the validity and efficiency of the proposed algorithm. At the end of the paper, a conclusion is presented. 


\section{VIBRO-ACOUSTIC ANALYSIS VIA FEM-BEM COUPLING}

\subsection{BOUNDARY ELEMENT FORMULATION FOR SOUND RADIATION}

Consider the following Helmholtz equation governing the acoustic wave domain:

$$
\nabla^{2} p(\mathbf{x})+k^{2} p(\mathbf{x})=0, \quad \forall \mathbf{x} \in \Omega
$$

where $\nabla^{2}$ is the Laplace operator, $p(\mathbf{x})$ is the acoustic pressure at field point $\mathbf{x}, k=\omega / c$ is the wave number, $\omega$ is the angular frequency, and $c$ is the wave speed in the acoustic medium $\Omega$. The common time-dependent term $e^{-i \omega t}(i=\sqrt{-1})$ is omitted throughout this paper. Applying Green's second theorem to the Helmholtz equation yields the following integral equation:

$$
p(\mathbf{x})+\int_{\Gamma} \frac{\partial G(\mathbf{x}, \mathbf{y})}{\partial \vec{n}(\mathbf{y})} p(\mathbf{y}) \Gamma(\mathbf{y})=\int_{\Gamma} G(\mathbf{x}, \mathbf{y}) \omega \rho v_{f}(\mathbf{y}) \Gamma(\mathbf{y})+p^{\text {inc }}(\mathbf{x})
$$

where $\mathbf{x}$ is the field point, $\mathbf{y}$ is the source point, $\Gamma=\partial \Omega$ is the boundary of domain $\Omega, \vec{n}(\mathbf{y})$ denotes the outward normal vector at point $\mathbf{y}, v_{f}(\mathbf{y})$ is the normal fluid particle velocity related to sound pressure via the Euler equation in the frequency domain.

$$
v_{f}(\mathbf{x})=\frac{1}{\omega \rho} \frac{\partial p(\mathbf{x})}{\partial \vec{n}(\mathbf{x})}
$$

$G(\mathbf{x}, \mathbf{y})$ is the Green's function. For three-dimensional acoustic problems, the fundamental solution is given as:

$$
G(\mathbf{x}, \mathbf{y})=\frac{e^{\mathrm{ik}|\mathbf{x}-\mathbf{y}|}}{4 \pi|\mathbf{x}-\mathbf{y}|}
$$

Shifting from point approach to the boundary leads to the Kirchhoff-Helmholtz boundary integral equation referred to as CBIE:

$$
c(\mathbf{x}) p(\mathbf{x})+\int_{\Gamma} \frac{\partial G(\mathbf{x}, \mathbf{y})}{\partial n(\mathbf{y})} p(\mathbf{y}) \Gamma(\mathbf{y})=\int_{\Gamma} G(\mathbf{x}, \mathbf{y}) \omega \rho v_{f}(\mathbf{y}) \Gamma(\mathbf{y})+p^{\text {inc }}(\mathbf{x})
$$

where the coefficient $c(\mathbf{x})$ is $1 / 2$ if the boundary is smooth around the source point $\mathbf{x}$, $G(\mathbf{x}, \mathbf{y})$ is the Green's function and $p^{\text {inc }}(\mathbf{x})$ is the pressure resulting from the incident wave in the $\mathrm{x}$-direction. Taking the derivative of CBIE with respect to the outward normal at point $\mathbf{x}$ if the boundary is smooth around $\mathbf{x}$, the hyper-singular boundary integral equation (HBIE) for acoustic problems is obtained as:

$$
\frac{1}{2} \frac{\partial p(\mathbf{x})}{\partial \vec{n}(\mathbf{x})}+\int_{\Gamma} \frac{\partial^{2} G(\mathbf{x}, \mathbf{y})}{\partial \vec{n}(\mathbf{x}) \partial \vec{n}(\mathbf{y})} p(\mathbf{y}) \Gamma(\mathbf{y})=\int_{\Gamma} \frac{\partial G(\mathbf{x}, \mathbf{y})}{\partial \vec{n}(\mathbf{x})} \omega \rho v_{f}(\mathbf{y})+\frac{\partial p^{\mathrm{inc}}(\mathbf{x})}{\partial \vec{n}(\mathbf{x})}
$$

Apparently, Eq. (6) requires the normal vector at point $\mathbf{x}$, i.e., $\vec{n}(\mathbf{x})$, to be unique. In this case, the coefficient $c(\mathbf{x})$ keeps a constant of $1 / 2$. It is well-known that the implementation of a single Helmholtz BIE, i.e., CBIE or HBIE, may suffer from nonuniqueness for exterior boundary- 
value problems. However, Zheng et al. [28] recently found that the nonuniqueness issue also exists for interior problems. Because the fictitious eigenfrequency of the BEM based on the CBIE or HBIE has very small imaginary parts, the troubles to interior acoustic radiation and scattering problems might appear. To overcome this key issue, the Burton-Miller formulation [29] is employed. In Burton and Miller formulation, a linear combination of CBIE and HBIE is used to give a unique solution for all frequencies as follows [29]:

$$
\begin{aligned}
& \frac{1}{2} p(\mathbf{x})+\frac{\alpha}{2} \omega \rho v_{f}(\mathbf{x})+\int_{\Gamma} \frac{\partial G(\mathbf{x}, \mathbf{y})}{\partial \vec{n}(\mathbf{y})} p(\mathbf{y}) \Gamma(\mathbf{y})+\alpha \int_{\Gamma} \frac{\partial^{2} G(\mathbf{x}, \mathbf{y})}{\partial \vec{n}(\mathbf{x}) \partial \vec{n}(\mathbf{y})} p(\mathbf{y}) \Gamma(\mathbf{y}) \\
= & \int_{\Gamma} G(\mathbf{x}, \mathbf{y}) \omega \rho v_{f}(\mathbf{y}) \Gamma(\mathbf{y})+\alpha \int_{\Gamma} \frac{\partial G(\mathbf{x}, \mathbf{y})}{\partial \vec{n}(\mathbf{x})} \omega \rho v_{f}(\mathbf{y}) \Gamma(\mathbf{y})+p^{\text {inc }}(\mathbf{x})+\alpha \frac{\partial p^{\text {inc }}(\mathbf{x})}{\partial \vec{n}(\mathbf{x})}
\end{aligned}
$$

where $\alpha$ is the coupling constant that is complex with non-vanishing imaginary part $(\operatorname{Im}(\alpha) \neq 0)$. Regarding the formulations in the current work, the optimal coupling parameter is $-i / k$. Note that the optimal coupling parameter varies when using different timedependence terms and fundamental functions as discussed by Marburg [31]. Before discretization, we introduce the admittance boundary condition to simulate the pressure attenuation caused by porous absorbing materials, as follows:

$$
v_{f}(\mathbf{x})-v_{s}(\mathbf{x})=Y(\mathbf{x}) p(\mathbf{x}), \quad \forall \mathbf{x} \in \Gamma
$$

where $v_{S}$ is the structural particle velocity usually provided as boundary conditions. For example, $v_{S}$ will be zero if the structure is rigid. $Y$ is the surface admittance. Considering sound-absorbing material attached to the surface of the structures, the absorption coefficient of acoustic conductance needs to be studied. Disregarding the physical parameters of the sound-absorbing material, such as thickness, and considering the admittance boundary condition and the continuity boundary condition [32], $Y$ meets:

$$
\frac{\partial p(\mathbf{x})}{\partial \vec{n}(\mathbf{x})}=i k \beta p(\mathbf{x})=Y(\mathbf{x}) p(\mathbf{x})
$$

in which $\beta$ is the media acoustic admittance [33]. For algorithm research, $\beta$ is set to be 0 for rigid-backing, and is set to 1 for a full sound-absorbing effect which could be regarded as nonreflecting boundary conditions [34].

Note that we use the admittance boundary condition instead of the commonly-used impedance boundary condition to avoid using an infinite impedance value to simulate a sound rigid case.

Discretizing of Burton-Miller formulation by collocation method and incorporating boundary condition (8) lead to the following linear system of equation:

$$
(\mathbf{H}-\mathbf{G Y}) \mathbf{p}=\mathbf{G v}_{S}+\mathbf{p}^{\text {inc }}
$$

where $\mathbf{H}$ and $\mathbf{G}$ are the BEM coefficient matrices usually fully populated, $\mathbf{p}$ and $\mathbf{v}_{S}$ are the vector forms of sound pressure $p$ and structural particle velocity $v_{S}, \mathbf{p}^{\text {inc }}$ is the vector resulting from an incident wave. Strong singular and hypersingular integrals exist in BurtonMiller formulation, respectively. In this research, they are evaluated directly when constant elements are employed to discretize the boundary [35]. Another difficulty in solving Eq. (10) consists of the non-symmetric and dense matrices $\mathbf{H}$ and $\mathbf{G}$. This makes the method 
unsuitable for the large-scale problem due to its high computational costs. For the computational efforts to be reduced, the generalized minimal residual (GMRES) method is applied to solve the equation using the fast multipole method (FMM). The FMM decompose the coefficient matrix $\mathbf{H}$ or $\mathbf{G}$ into several much smaller matrices, obviously reducing the storage and accelerating the matrix-vector multiplications. For details to solve the above equation based on the FMM, readers are referred to Ref. [36].

\subsection{FINITE ELEMENT FORMULATION FOR STRUCTURAL VIBRATIONS}

The harmonic structural vibrations is expressed by the following:

$$
\left(\mathbf{K}-i \omega \mathbf{D}-\omega^{2} \mathbf{M}\right) \mathbf{u}=\mathbf{f}
$$

where $\mathbf{K}$, $\mathbf{D}$, and $\mathbf{M}$ represent the stiffness matrix, damping matrix, and mass matrix, respectively. u denotes the nodal displacement vector. $\mathbf{f}$ is the external load vector, that consists of two parts. One is the structural load; another is the acoustic pressure load from the fluid. Thus,

$$
\mathbf{f}=\mathbf{f}_{s}+\mathbf{T}_{s f} \mathbf{p}
$$

where $\mathbf{T}_{s f}$ is the transformation matrix with $s$ structural DOFs and $f$ fluid DOFs.

Using Eq. (8) and Eq. (10), we obtain the following formula:

$$
\mathbf{v}_{f}=-\mathrm{i} \omega \Theta^{-1} \mathbf{T}_{f s} \mathbf{u}+\mathbf{Y p}
$$

where $\mathbf{T}_{f s}$ is coupling matrix, satisfying $\mathbf{T}_{s f}=\mathbf{T}_{s f}^{T} \cdot \Theta$ is the boundary mass matrix that is symmetric.

By combining Eq. (10) and Eq. (11), we derive the following coupled system equations:

$$
\left[\begin{array}{cc}
\mathbf{K}-\mathrm{i} \omega \mathbf{D}-\omega^{2} \mathbf{M} & -\mathbf{T}_{s f} \\
\mathrm{i} \omega \mathbf{G} \Theta^{-1} \mathbf{T}_{f s} & \mathbf{H}-\mathbf{G Y}
\end{array}\right]\left[\begin{array}{l}
\mathbf{u} \\
\mathbf{p}
\end{array}\right]=\left[\begin{array}{c}
\mathbf{f}_{s} \\
\mathbf{p}^{\text {inc }}
\end{array}\right]
$$

By employing the Schur complement to eliminate the pressure unknowns, we obtain:

$$
\left[\mathbf{K}-\mathrm{i} \omega\left(\mathbf{D}+\mathbf{D}_{f}\right)-\omega^{2}\left(\mathbf{M}+\mathbf{M}_{f}\right)\right] \mathbf{u}=\mathbf{f}_{s}
$$

with:

$$
\mathbf{D}_{f}=-\operatorname{Re}\left[\mathbf{T}_{s f}(\mathbf{H}-\mathbf{G Y})^{-1} \mathbf{G} \Theta^{-1} \mathbf{T}_{f s}\right], \mathbf{M}_{f}=-\frac{\mathrm{i}}{\omega} \operatorname{Im}\left[\mathbf{T}_{s f}(\mathbf{H}-\mathbf{G Y})^{-1} \mathbf{G} \Theta^{-1} \mathbf{T}_{f s}\right]
$$

in which $\operatorname{Re} 0$ and $\operatorname{Im}()$ means taking the real part and the imaginary part of the vector 0 , respectively. Thus, the acoustic loads on the structure can be regarded as a combination of added mass $\mathbf{M}_{f}$ and added damping $\mathbf{D}_{f}$ that are frequency-dependent. When optimizing the admittance condition, i.e., the admittance matrix $\mathbf{Y}$, both the added mass and added damping are influenced. Thus, the vibration and sound radiation can be somewhat tuned by adjusting the admittance, more precisely in this paper, by adjusting the distribution of the absorbing layer over the outer surface. Here, the coupled system is solved by employing the Schur complement to eliminate the displacement unknowns and then solving the pressures as: 


$$
\left[\mathbf{H}-\mathbf{G}\left(\mathbf{Y}-\mathbf{Y}_{c}\right)\right] \mathbf{p}=\mathbf{G W \mathbf { f } _ { s }}+\mathbf{p}^{\text {inc }}
$$

where $\mathbf{W}=\mathrm{i} \omega \Theta^{-1} \mathbf{T}_{f s}\left(\mathbf{K}-\mathrm{i} \omega \mathbf{D}-\omega^{2} \mathbf{M}\right)^{-1}$ and $\mathbf{Y}_{c}=\mathbf{W} \mathbf{T}_{s f}$ can be regarded as the coupling admittance derived from the structure part [37]. Compared with Eq. (14), the reduced equation above owns a much smaller condition number, yielding a faster convergence in iterative solution. After obtaining the pressures on the coupling interface, the displacements is easily computed based on a sparse direct solver such as PARDISO [38].

\section{SENSITIVITY ANALYSIS}

The objective of the optimization is to find the best design parameters that define the performance of the structure in some aspects under certain constraints. Design sensitivity analysis is a procedure for calculating the gradients of defined objective functions. The obtained gradients are used to determine the direction for searching the optimum values of the design variables.

In such acoustic optimization, the sensitivity of sound pressure at the predefined position or sound power is the key point that shows the different variables' effect on the objective function, i.e., sound pressures on the coupling interface and displacements. Hence, the derivatives of coupled Eq. (17) could be expressed as:

$$
\begin{aligned}
& {\left[\mathbf{H}-\mathbf{G}\left(\mathbf{Y}-\mathbf{Y}_{c}\right)\right] \dot{\mathbf{p}}+\left[\dot{\mathbf{H}}-\dot{\mathbf{G}}\left(\mathbf{Y}-\mathbf{Y}_{c}\right)-\mathbf{G}\left(\dot{\mathbf{Y}}-\dot{\mathbf{Y}}_{c}\right)\right] \mathbf{p}} \\
& =\dot{\mathbf{G}} \mathbf{W} \mathbf{f}_{s}+\mathbf{G} \dot{\mathbf{W}}_{s}+\mathbf{G W} \dot{\mathbf{f}}_{s}+\dot{\mathbf{p}}
\end{aligned}
$$

where the upper $\operatorname{dot}()$ means the differentiation concerning the design variable.

Eq. (18) is transformed to:

$$
\begin{aligned}
& {\left[\mathbf{H}-\mathbf{G}\left(\mathbf{Y}-\mathbf{Y}_{c}\right)\right] \dot{\mathbf{p}}} \\
& =-\dot{\mathbf{H}} \mathbf{p}+\dot{\mathbf{G}}\left(\mathbf{Y}-\mathbf{Y}_{c}\right) \mathbf{p}+\mathbf{G}\left(\dot{\mathbf{Y}}-\dot{\mathbf{Y}}_{c}\right) \mathbf{p}+\dot{\mathbf{G}} \mathbf{W} \mathbf{f}_{s}+\mathbf{G} \dot{\mathbf{W}} \mathbf{f}_{s}+\mathbf{G W} \dot{\mathbf{f}}_{s}+\mathbf{p}^{\dot{\mathrm{inc}}} \\
& =\dot{\mathbf{G}}\left[\left(\mathbf{Y}-\mathbf{Y}_{c}\right) \mathbf{p}+\mathbf{W} \mathbf{f}_{s}\right]+\mathbf{G}\left[\left(\dot{\mathbf{Y}}-\dot{\mathbf{Y}}_{c}\right) \mathbf{p}+\dot{\mathbf{W}} \mathbf{f}_{s}+\mathbf{W} \dot{\mathbf{f}}_{s}\right]-\dot{\mathbf{H} p}+\mathbf{p}^{\dot{\mathrm{inc}}}
\end{aligned}
$$

And simplified to be:

$$
\left[\mathbf{H}-\mathbf{G}\left(\mathbf{Y}-\mathbf{Y}_{c}\right)\right] \dot{\mathbf{p}}=\dot{\mathbf{G}} \lambda_{1}+\mathbf{G} \lambda_{2}-\dot{\mathbf{H}} \mathbf{p}+\mathbf{p}^{\dot{\text { inc }}}
$$

where vectors $\lambda_{1}$ and $\lambda_{2}$ are expressed as follows: 


$$
\begin{aligned}
& \lambda_{1}=\left(\mathbf{Y}-\mathbf{Y}_{c}\right) \mathbf{p}+\mathbf{W} \mathbf{f}_{s} \\
& \lambda_{2}=\left(\dot{\mathbf{Y}}-\dot{\mathbf{Y}}_{c}\right) \mathbf{p}+\dot{\mathbf{W}} \mathbf{f}_{s}+\mathbf{W} \dot{f}_{s} \\
& \dot{\mathbf{W}}=\mathrm{i} \omega\left(\dot{\Theta}^{-1} \mathbf{T}_{f_{S}} \mathbf{A}^{-1}+\Theta^{-1} \dot{\mathbf{T}} f_{s} \mathbf{A}^{-1}+\Theta^{-1} \mathbf{T}_{f_{s}} \dot{\mathbf{A}}^{-1}\right) \\
& \mathbf{A}=\mathbf{K}-i \omega \mathbf{D}-\omega^{2} \mathbf{M}
\end{aligned}
$$

Substitute all boundary acoustic pressure values into Eq. (20), we obtain the computational solution of matrix-vector products on the right side of Eq. (20). The value of vector $\lambda_{1}$ and $\lambda_{2}$ are computed by Eq. (21). The matrices $\dot{\mathbf{Y}}, \dot{\mathbf{Y}}_{c}, \dot{\Theta}^{-1}, \dot{\mathbf{T}}_{f s}$ are solved easily when the boundary is determined. The matrix $A$ is the coefficient matrix of the finite element model derived by the finite element method. It is time-consuming and poor efficiency to compute matrix $\dot{\mathbf{A}}^{-1}$ by operating the matrix A directly. To overcome this problem, an indirect method is proposed by solving linear equations. The details are as follows:

$$
\dot{\mathbf{A}}^{-1}=-\mathbf{A}^{-1} \dot{\mathbf{A}} \mathbf{A}^{-1}
$$

Substituting Eq. (22) into the third formulation in Eq. (21), we find the solution of $\mathbf{W y}$ by solving the equation $\mathbf{A x}=\mathbf{y}$. Finally, all the coefficient matrix of Eq. (20) is derived, and the sensitivities of design variables are then computed.

\section{NUMERICAL TESTS}

To investigate the validity and applicability of the developed approach, some numerical tests are performed. The GMRES implementation without preconditioning is employed to solve all linear systems of Eq. (17), and the tolerance of the GMRES solver is set to be 1.0*e-4. In all the examples, the constant boundary elements are used in the acoustic analysis, and the 8-node shell element (SHELL281 in ANSYS) is used in finite element analysis. All computations are implemented in an in-house Fortran 95/2003 code and carried out on a desktop PC with an Intel Core i7 CPU and 32 GB RAM.

\subsection{SCATTERING OF A THIN SPHERICAL SHELL WITH SOUND-ABSORBING MATERIAL}

In this section, the scattering sound field of an underwater thin spherical shell is analysed considering the action of sound-absorbing materials when plane waves are incident. The plane wave propagates in the positive direction along the $\mathrm{x}$-axis with an amplitude of $1.0 \mathrm{~Pa}$, as shown in Figure 1. Spherical shell radius $a=1.0 \mathrm{~m}$, radius to thickness ratio is 100:1, structural density $\rho=7860 \mathrm{~kg} / \mathrm{m}^{3}$, modulus of elasticity $E=210 \mathrm{GPa}$, and Poisson's ratio $v=0.3$. The density of the water body is $\rho=1000 \mathrm{~kg} / \mathrm{m}^{3}$, and the wave velocity in water $c=1482 \mathrm{~m} / \mathrm{s}$. The part of the structure is discretized with the 8-node quadrilateral finite element SHELL281, and the part of the fluid with the constant boundary element. 


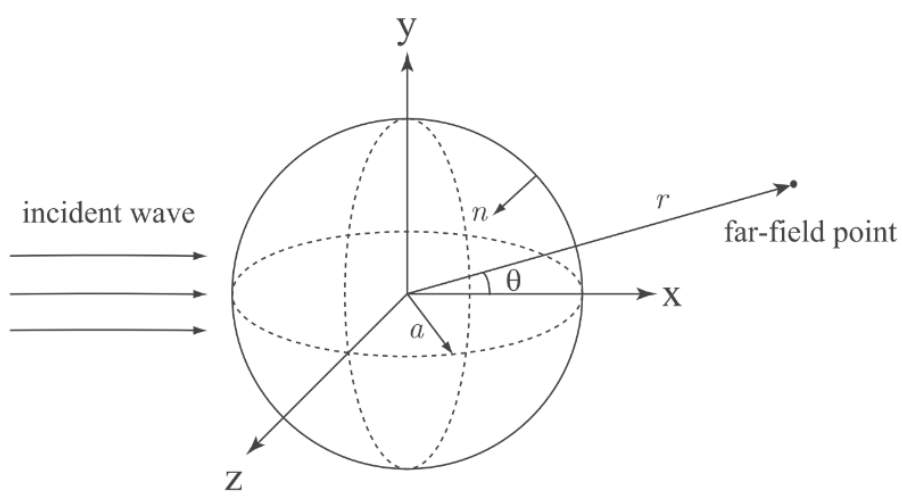

Fig. 1 Scattering from a spherical shell with a radius a

Figure 2 shows the variation of the scattering sound pressure with frequency at the point $(2 a$, 0,0 ). It can be seen from the figure that the value of sound pressure in the low-frequency band is low, that in the high-frequency band is high, and at the natural frequency of the structural vibration there is a sudden jump. In addition, the numerical results are very consistent with the analytical results verifying the correctness of the algorithm in this paper.

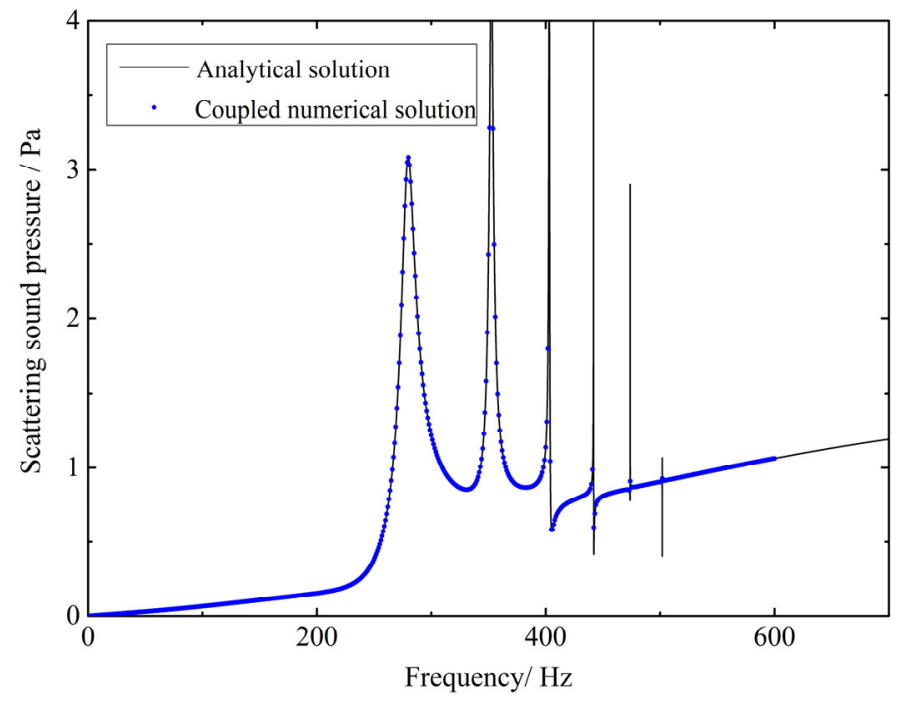

Fig. 2 Scattering sound pressure at point $(2 a, 0,0)$

Figure 3 shows the relationship between the relative error and the number of DOFs at $300 \mathrm{~Hz}$. Observing the graph, we notice that as the number of DOFs increases, the relative error between the numerical solution and the analytical solution decreases rapidly, proving the convergence and correctness of the numerical calculation. 


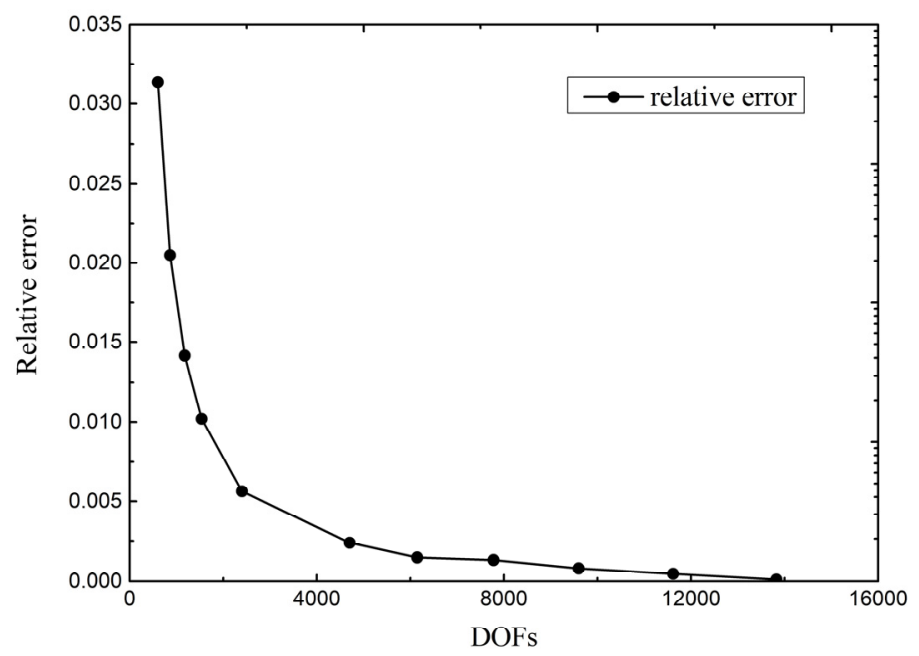

Fig. 3 Relative errors of the scattering sphere example

The incident wave frequency is assumed to be $100 \mathrm{~Hz}$, and the medium admittance $\beta$ is taken as 0 and 1, respectively. Figure 4 shows the sound pressure level at a series of points on the ring with a $2 a$ radius in the XOY plane. As seen from the figure, when the medium admittance $\beta$ is 0 , the numerical and analytical solutions are in good agreement. When $\beta$ is 1 , the sound pressure level at each calculation point is significantly lower than the result of $\beta=0$, showing that the use of sound-absorbing material effectively reduces the radiation or scattering noise of structural vibration.

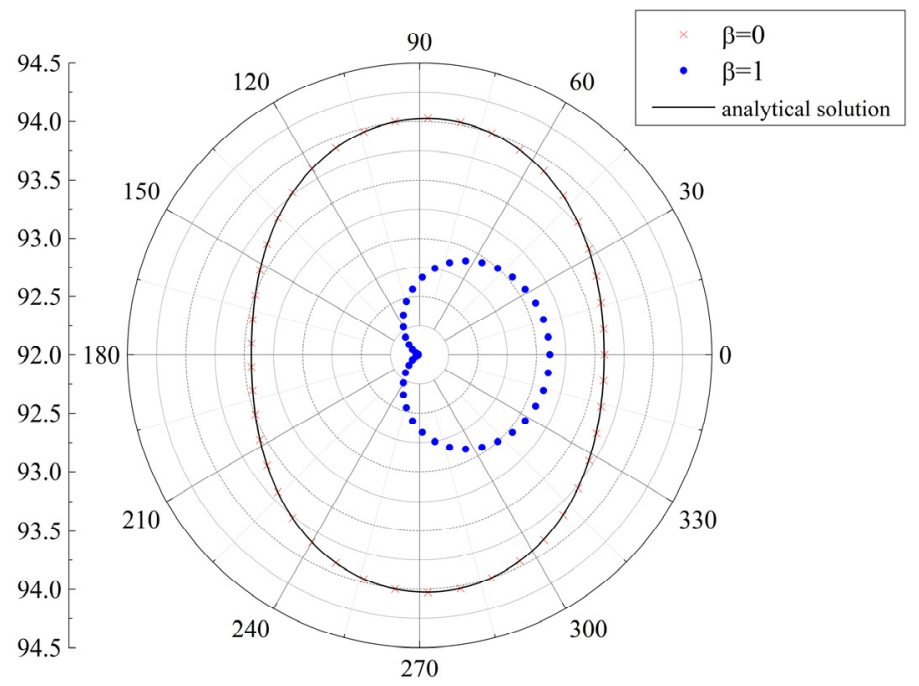

Fig. 4 Coupling sound pressure level at ring with radius $2 a$

The iterative solution of Eq. (17) is determined by the GMRES method, the convergence accuracy is set to $1.0 \times 10^{-4}$, and the variation of the number of iterations with frequency is investigated. Figure 5 shows the number of iterations required for convergence at different frequencies where "rigid solution" represents the number of iterations of rigid scattering without considering acoustic-vibration coupling and "elastic solution" represents the result of an elastic structure considering acoustic-vibration coupling. It is noticeable from the results of rigid scattering that as the calculation frequency increases, the number of iterations also gradually increases. Examining the iteration results of the elastic structure, we see that as the 
frequency increases, the number of iterations shows an overall upward trend; but at the natural frequency of the structure, the number of iterations increases in a leaping manner. In addition, comparing the two curves, it is found that when calculating the same frequency, the number of iterations when considering coupling is higher than that without considering it. This is because the vibration calculation of the coupled elastomer structure is more complex, and the convergence deteriorates. Therefore, it is important to develop suitable preprocessing methods to reduce the number of iterations required to solve the coupled equations, especially for large-scale practical problems.

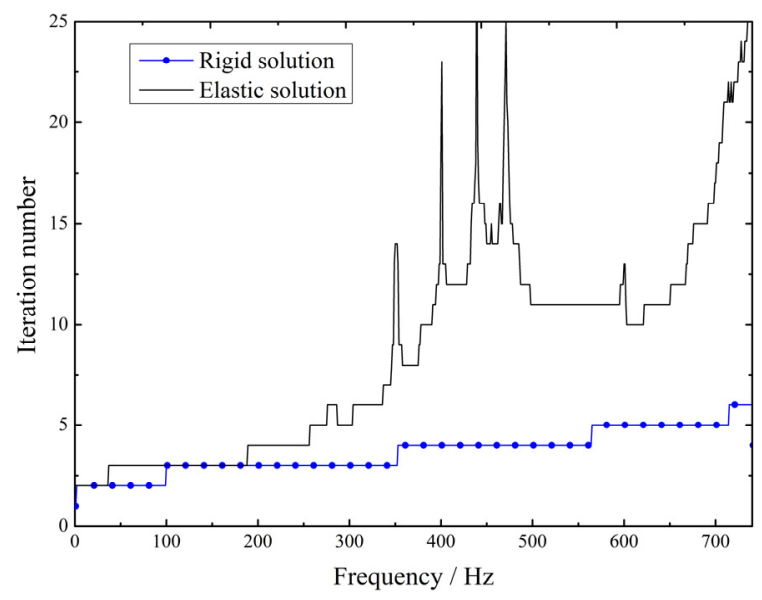

Fig. 5 Iteration number

The calculation frequency is now assumed to be as $50 \mathrm{~Hz}$. Figure 6 compares the calculation time spent for different algorithms. Therein, "rigid solution" and "accelerated rigid solution" refer to the time required to calculate the sound pressure of the rigid ball using CBEM and Wideband FMBEM; "Elastic solution" and "accelerated elastic solution" refer to the calculation of elasticity using Conventional BEM (CBEM) and Wideband Fast Multipole BEM (FMBEM), respectively. As seen from the figure, the number of DOFs increases, the acceleration effect of Wideband FMBEM becomes more obvious. In addition, coupled analysis takes more time than uncoupled, because, in the coupled analysis process, each step requires the iterative solution of the $\boldsymbol{G W C}_{s f} \boldsymbol{p}_{k}$ term (the $k^{\text {th }}$ iteration substep) and $\boldsymbol{G} \boldsymbol{W} \boldsymbol{f}_{s}$ term in Eq. (17). Figure 5 shows that the number of iterations of elastic coupling convergence is much greater than the number of rigid analyses, so the coupling analysis takes more time.

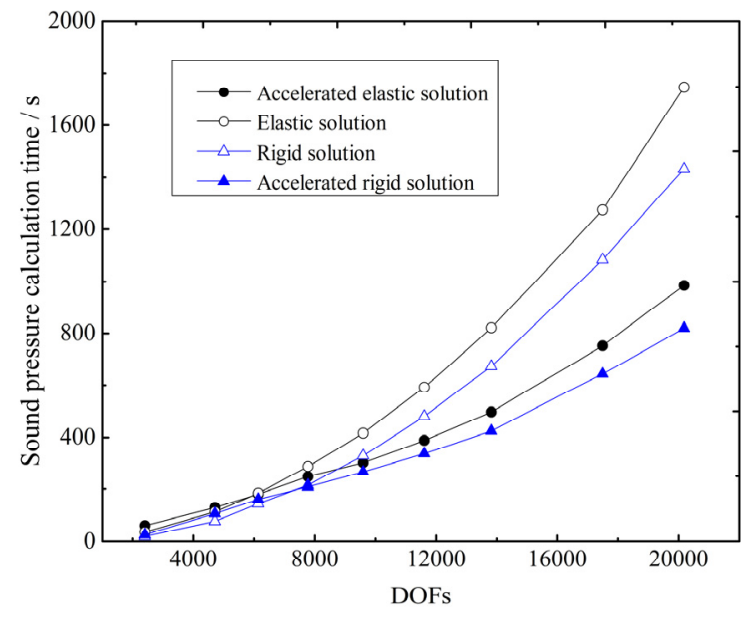

Fig. 6 Impact of element number on CPU time 


\subsection{THIN SPHERICAL SHELL WITH SOUND-ABSORBING MATERIAL UNDER SINGLE- POINT EXCITATION}

The concentrated force load $F$ acts on the point $(a, 0,0)$ of the spherical shell in the negative $\mathrm{x}$ axis direction. Considering the balance between the viscous resistance and the concentration force of the water-on-structure, the structure could stay in place. Figure 7 shows the comparison between the numerical and analytical solutions of the radiated sound power on the structure surface [13]. It is noticeable from the results in the figure that the numerical solution and the analytical solution are in good agreement, which again verifies the correctness of the algorithm and program used in this paper. The calculated frequency is now $200 \mathrm{~Hz}$, and the sound power on any spherical surface surrounding the vibration structure is investigated. Figure 8 shows how the real and imaginary parts of the polyphony power vary with the radius of the calculated surface. It can be seen from the figure that the imaginary part of the complex sound power only works in the near field, and as the radius of the calculated surface increases, the absolute value of the imaginary part decreases rapidly. With the increase of the radius, the real part of the complex sound power, that is, the radiated sound power is not lost. In fact, the radiated acoustic power characterizes the acoustic energy of radiation entering the fluid, so it is of great practical engineering significance to investigate the radiated sound power.

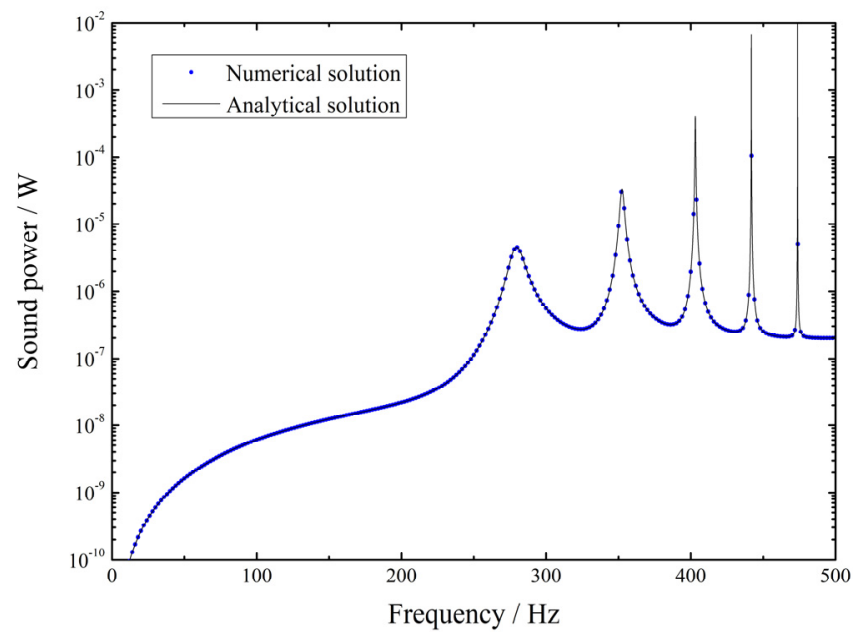

Fig. 7 Sound power on structure surface

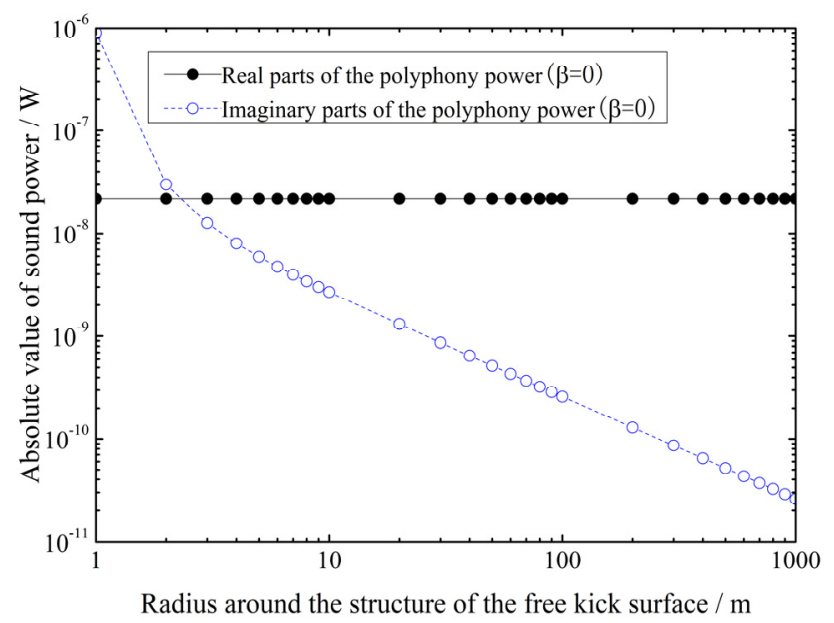

Fig. 8 Impact of radius of the computed spherical surface on sound power 
The frequency of the incident wave is now $200 \mathrm{~Hz}$. Figure 9 shows the change of the sound pressure at the point $(2 a, 0,0)$ with the admittance value of the medium. As seen from the figure, the admittance of the medium increases, the sound pressure value at the calculation point gradually decreases, and it is noticeable that the sound absorption effect increases as the admittance value of the medium increases. In addition, the coupled solution is significantly lower than the uncoupled solution, and the effect of water on the vibration of the elastic body cannot be ignored when calculating the sensitivity of the scattering sphere's sound pressure at point $(2 a, 0,0)$ concerning thickness. Figure 10 shows the analytical and numerical solutions for the sensitivities of the scattering sound pressure concerning thickness $t$ of the sphere. The numerical solution is basically consistent with the analytical solution, proving the validity of the algorithm.

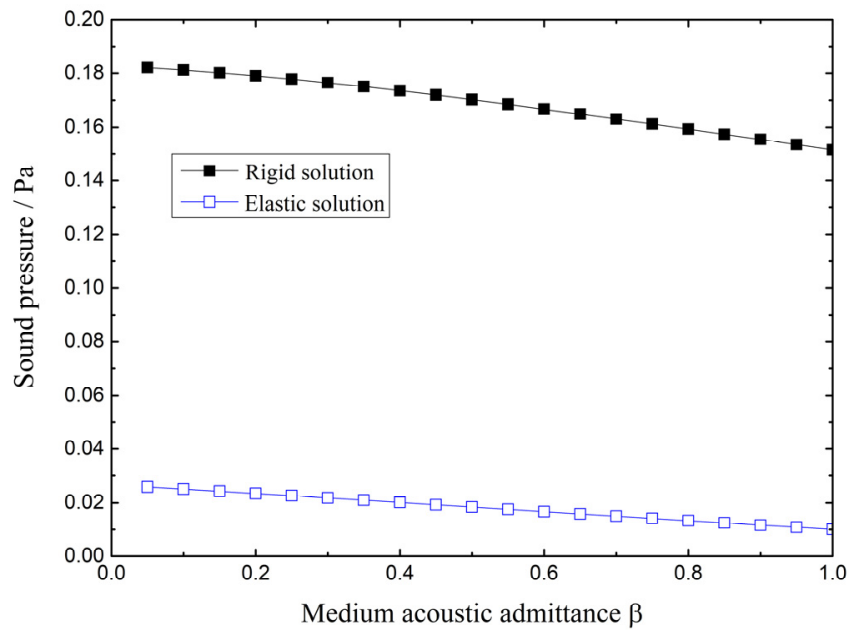

Fig. 9 Sound pressure at point $(2 a, 0,0)$

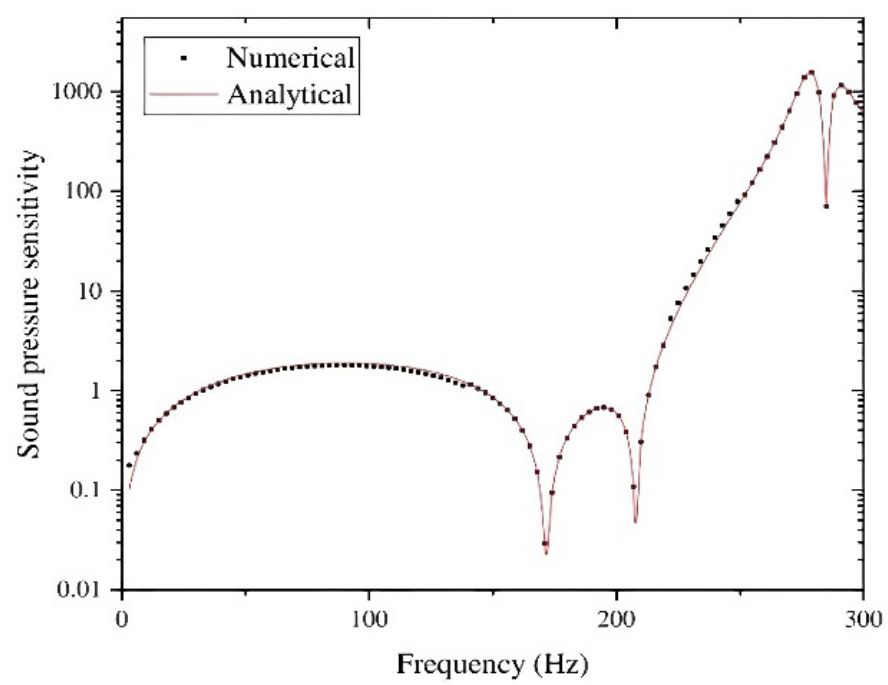

Fig. 10 Sensitivities of scattering sound pressure at point $(2 a, 0,0)$ with respect to thickness 


\subsection{SCATTERING FROM A SUBMARINE MODEL}

This section deals with the scattering sound field of the underwater submarine model considering the sound-absorbing materials when plane waves are incident. The plane wave propagates positively along the $\mathrm{x}$-axis, and the incident wave amplitude is $1.0 \mathrm{~Pa}$. The model adopts the general model BeTSSi-Sub provided at the 2002 World Digital Simulation Conference. The thickness of the model is $0.05 \mathrm{~m}$. For detailed geometric parameters, please refer to Ref. [39]. The origin of the coordinate is at the center of the cross section of the circle connecting the bow and the hull, and the positive x-axis direction is from the bow to the bow. The complete calculation process implies using CATIA software to establish the geometric model, importing ANSY to mesh, extracting the overall mass matrix, stiffness matrix, and element knots relationship, importing the Fortran program to calculate the sound field and analyse the results. The Fortran uses the FMBEM method to accelerate calculation. If it is not accelerated, the convergence process takes a lot of time and analysis cannot be performed normally. In addition, ANSYS uses an 8-node structural shell element SHELL281 to divide the triangular grid, the total number of elements is 27020, and the grid division is shown in Figure 11.

The calculation point is at $(60,0,0)$, and the relationship between the scattering sound pressure at the calculation point and the admittance of the medium is investigated. Figure 12 shows the calculation result without considering the vibration coupling, and Figure 13 is the result considering the vibration coupling. Observing Figure 12, it can be found that with the increase of the admittance of the medium, at the same frequency, the sound pressure value gradually decreases, indicating that the higher the admittance value of the medium, the better the sound absorption effect without considering the vibration coupling.

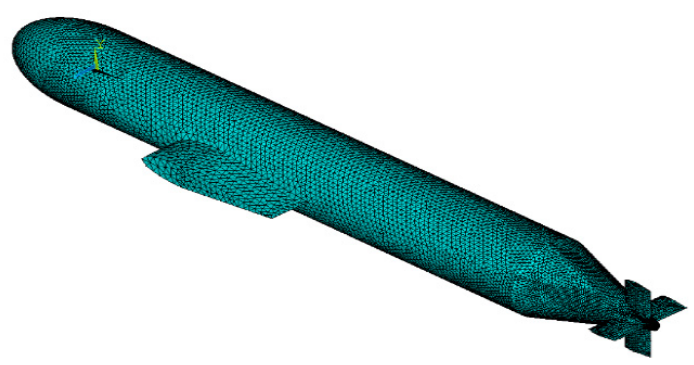

Fig. 11 Elements of a simple BeTSSi-Sub model

In Figure 13, the sound pressure value at low frequencies is found to be very small. The sound pressure value increases rapidly and stabilizes with increasing frequency. There is also a sudden jump near the natural frequency of the coupling structure. This kind of damping effect, in the vicinity of the natural frequency of the coupling structure, hinders the change of sound pressure, and the higher the admittance of the medium, the better the blocking effect. In addition, the calculation result considering acoustic-vibration coupling is obviously different from the result without considering acoustic-vibration coupling, indicating that the effect of water on the vibration of the elastic body cannot be ignored. 


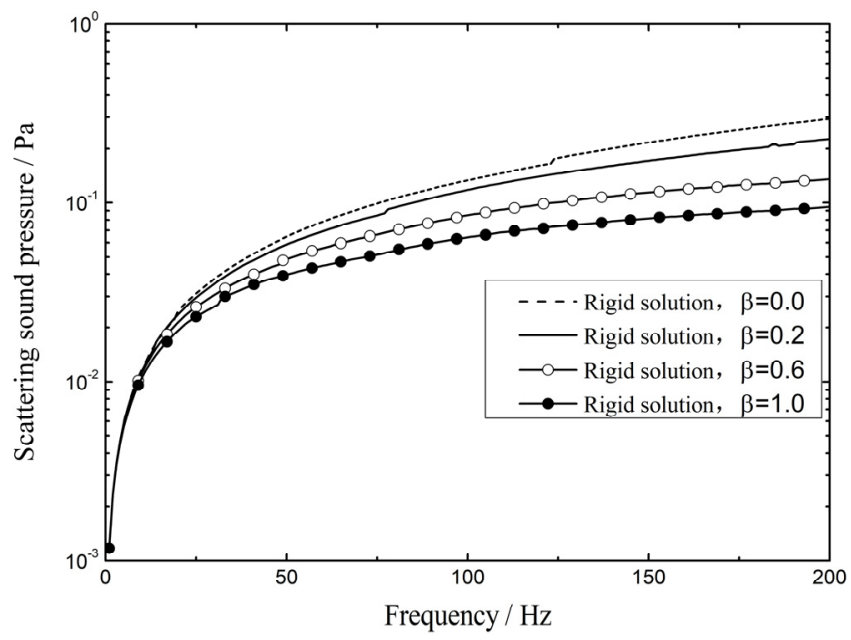

Fig. 12 Uncoupling sound pressure at point $(60,0,0)$

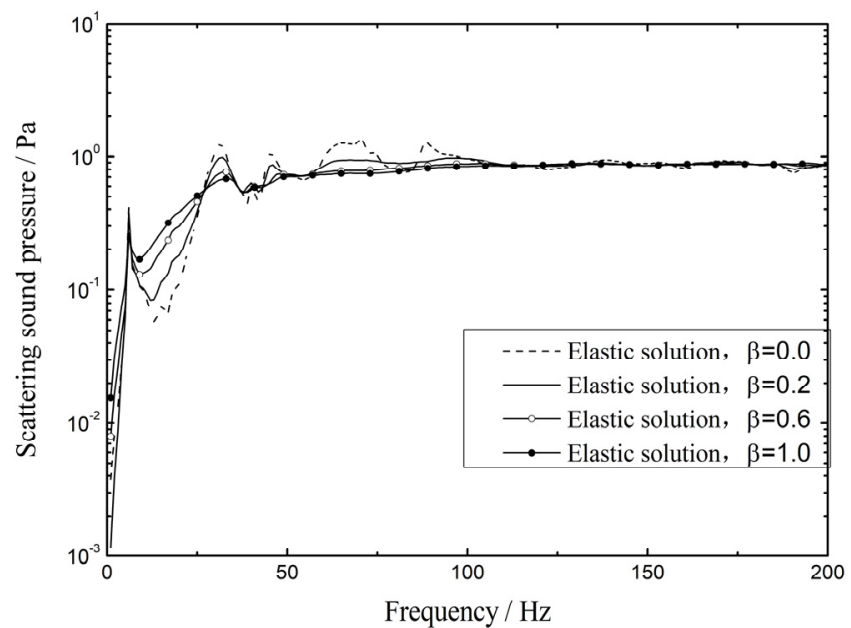

Fig. 13 Coupling sound pressure at point $(60,0,0)$

\section{CONCLUSION}

In this paper, the coupled FEM/Wideband FMBEM method is used to quickly analyse the radiation and scattering sound field of underwater elastomer structures. The sound power of structural vibration radiated when considering the coupling of sound and vibration is calculated, and calculation expressions of the radiated sound power of the structure surface and any surrounding structure surface in the fluid are derived, respectively. The media admittance parameters are introduced to preliminary study the effect of sound-absorbing materials on the vibration radiation and scattering sound field distribution of underwater elastic structures. The more detailed physical parameters of sound-absorbing materials will be considered in subsequent research, while the changes on the structure mass matrix and stiffness matrix will be derived to further study the effects of sound-absorbing materials on the underwater acoustic-vibration coupling problem. In this paper, the correctness and effectiveness of the algorithm are verified by an underwater spherical shell model with analytical solutions and further applied to the analysis of the underwater fluid-structure coupling of the BeTSSi-Sub example. At the same time, the method in this paper improves the 
computational efficiency of the coupling problem and provides support for large-scale computing.

\section{REFERENCES}

[1] G.C. Everstine, F.M. Henderson, Coupled finite element/boundary element approach for fluid-structure interaction, Journal of the Acoustical Society of America, Vol. 87, No. 5, pp. 1938-1947, 1990. https://doi.org/10.1121/1.399320

[2] H. Zheng, G.R. Liu, J.S. Tao, K.Y. Lam, FEM/BEM analysis of diesel piston-slap induced ship hull vibration and underwater noise, Applied Acoustics, Vol. 62, No.4, pp. 341-358, 2001. https://doi.org/10.1016/S0003-682X(00)00046-3

[3] Q. Zhou, P. Joseph, FEM/BEM A numerical method for the calculation of dynamic response and acoustic radiation from an underwater structure, Journal of Sound and Vibration, Vol. 283, No. 3, pp. 853-873, 2005. https://doi.org/10.1016/i.jsv.2004.05.028

[4] S. Merz, R. Kinns, N. Kessissoglou, FEM/BEM Structural and acoustic responses of a submarine hull due to propeller forces, Journal of Sound and Vibration, Vol. 325, No. 1, pp. 266-286, 2009. https://doi.org/10.1016/i.jsv.2009.03.011

[5] H. Peters, N. Kessissoglou, S. Marburg, FEM/BEM Modal decomposition of exterior acoustic-structure interaction, Journal of the Acoustical Society of America, Vol. 133, No. 5, pp. 2668-2677, 2013. https://doi.org/10.1121/1.4796114

[6] D. Fritze, S. Marburg, H.J. Hardtke, FEM-BEM-coupling and structural-acoustic sensitivity analysis for shell geometries, advances in Analysis of Fluid Structure Interaction, Vol. 325, No. 1, pp. 143-154, 2005. https://doi.org/10.1016/i.compstruc.2004.05.019

[7] L.L. Chen, H. Lian, Z. Liu et.al, Structural shape optimization of three dimensional acoustic problems with isogeometric boundary element methods, Computer Methods in Applied Mechanics and Engineering, Vol. 355, No. 6, Article ID 06012, 2019.

https://doi.org/10.1016/j.cma.2019.06.012

[8] S. Marburg, Developments in structural-acoustic optimization for passive noise control, Archives of Computational Methods in Engineering, Vol. 9, No.4, pp. 291-370, 2002.

https://doi.org/10.1007/BF03041465

[9] H. Zheng, G. Liu, J. Tao et.al, FEM/BEM analysis of diesel piston-slap induced ship hull vibration and underwater noise, Applied Acoustics, Vol. 62, No. 4, Article ID 06012, 2001.

https://doi.org/10.1016/S0003-682X(00)00046-3

[10] Z.Y. Ai, Y.F. Chen, FEM-BEM coupling analysis of vertically loaded rock-socketed pile in multilayered transversely isotropic saturated media, Computers and Geotechnics, Vol. 120, No. 4, pp. 341-358, 2020. https://doi.org/10.1016/j.compgeo.2019.103437

[11] Z.Y. Ai, Y.C. Cheng, Extended precise integration method for consolidation of transversely isotropic poroelastic layered media, Computers Mathematics with Applications, Vol. 325, No. 1, pp. 1806-1818, 2014.

https://doi.org/10.1016/i.camwa.2014.10.010

[12] M. Junger, Acoustic fluid-elastic structure interactions: Basic concepts, Computers Structures, Vol. 65, No. 3, pp. 287-293, 1997. 
https://doi.org/10.1016/S0045-7949(96)00250-7

[13] D. Brunner, M. Junge, L. Gaul, A comparison of FE-BE coupling schemes for large-scale problems with fluid-structure interaction, International Journal for Numerical Methods in Engineering, Vol. 77, No. 5, pp. 664-688, 2009. https://doi.org/10.1002/nme.2412

[14] M. Fischer, L. Gaul, Application of the fast multipole bem for structural-acoustic simulations, Journal of Computational Acoustics, Vol. 13, No. 1, pp. 87-98, 2005.

https://doi.org/10.1142/S0218396X05002578

[15] H. Peters, S. Marburg, N. Kessissoglou, Structural-acoustic coupling on non-conforming meshes with quadratic shape functions, International Journal for Numerical Methods in Engineering, Vol. 91, No. 1, pp. 27-38, 2012. https://doi.org/10.1002/nme.4251

[16] A. Warszawski, D. Soares, W. Mansur, A FEM-BEM coupling procedure to model the propagation of interacting acoustic-acoustic/acoustic-elastic waves through axisymmetric media, Computer Methods in Applied Mechanics and Engineering, Vol. 197, No. 45, pp. 3828-3835, 2008. https://doi.org/10.1016/i.cma.2008.03.005

[17] S. Schneider, FE/FMBE coupling to model fluid-structure interaction, International Journal for Numerical Methods in Engineering, Vol. 76, No. 13, pp. 2137-2156, 2008.

https://doi.org/10.1002/nme.2399

[18] L. Shen, Y.J. Liu, An adaptive fast multipole boundary element method for threedimensional acoustic wave problems based on the burton-miller formulation, Computational Mechanics, Vol. 40, No. 3, pp. 461-472, 2007.

https://doi.org/10.1007/s00466-006-0121-2

[19] K. Yoshida, N. Nishimura, S. Kobayashi, Application of new fast multipole boundary integral equation method to crack problems in 3d, Engineering Analysis with Boundary Elements, Vol. 25, No. 4, pp. 239-247, 2001.

https://doi.org/10.1016/S0955-7997(01)00030-3

[20] H.T. Wang, Z.H. Yao, P.B. Wang, On the preconditioners for fast multipole boundary element methods for 2d multi-domain elastostatics, Engineering Analysis with Boundary Elements, Vol. 29, No. 7, pp. 673-688, 2005.

https://doi.org/10.1016/j.enganabound.2005.03.002

[21] C.J. Zheng, T. Matsumoto, T. Takahashi et.al, A wideband fast multipole boundary element method for three dimensional acoustic shape sensitivity analysis based on direct differentiation method, Engineering Analysis with Boundary Elements, Vol. 36, No. 3, Article ID 09001, 2012. https://doi.org/10.1016/j.enganabound.2011.09.001

[22] H.W. Cheng, W.Y. Crutchfield, Z. Gimbutas et.al, A wideband fast multipole method for the helmholtz equation in three dimensions, Journal of Computational Physics, Vol. 216, No. 1, Article ID 12001, 2006. https://doi.org/10.1016/i.jcp.2005.12.001

[23] N.A. Gumerov, R. Duraiswami, A broadband fast multipole accelerated boundary element method for the three dimensional helmholtz equation, Journal of the Acoustical Society of America, Vol. 125, No. 1, pp. 191-205, 2009. https://doi.org/10.1121/1.3021297

[24] W. Wolf, S.K. Lele, Wideband fast multipole boundary element method: Application to acoustic scattering from aerodynamic bodies, International Journal for Numerical Methods in Fluids, Vol. 67, No. 12, pp. 2108-2129, 2011. 


\section{https://doi.org/10.1002/fld.2486}

[25] L.L. Chen, H.B. Chen, C.J. Zheng et.al, Structural-acoustic sensitivity analysis of radiated sound power using a finite element/discontinuous fast multipole boundary element scheme, International Journal for Numerical Methods in Fluids, Vol. 82, No. 12, Article ID 4244, 2016. https://doi.org/10.1002/fld.4244

[26] L.L. Chen, S. Marburg, H.B. Chen et.al, An adjoint operator approach for sensitivity analysis of radiated sound power in fully coupled structural-acoustic systems, Journal of Computational Acoustics, Vol. 25, No. 1, Article ID 1750003, 2017.

https://doi.org/10.1142/S0218396X17500035

[27] L.L. Chen, C. Liu, W.C. Zhao et,al, An isogeometric approach of two dimensional acoustic design sensitivity analysis and topology optimization analysis for absorbing material distribution, Computer Methods in Applied Mechanics and Engineering, Vol. 336, No. 3, Article ID 03025, 2018. https://doi.org/10.1016/i.cma.2018.03.025

[28] C.J. Zheng, C.X. Bi, C.Z. Zhang et.al, Fictitious eigenfrequencies in the BEM for interior acoustic problems, Engineering Analysis with Boundary Elements, Vol. 104, No. 3 Article ID 03042, 2019. https://doi.org/10.1016/j.enganabound.2019.03.042

[29] A. Burton, G. Miller, The application of integral equation methods to the numerical solution of some exterior boundary-value problems, Physical and Engineering Sciences, Vol. 323, No. 1553, pp. 201-210, 1971. https://doi.org/10.1098/rspa.1971.0097

[30] L.L. Chen, C. Lu, H.J. Lian et.al, Acoustic topology optimization of sound absorbing materials directly from subdivision surfaces with isogeometric boundary element methods, Computer Methods in Applied Mechanics and Engineering, Vol. 362, Article ID 112806, 2020. https://doi.org/10.1016/i.cma.2019.112806

[31] S. Marburg, The Burton and Miller Method: Unlocking Another Mystery of Its Coupling Parameter, Journal of Computational Acoustics, Vol. 24, No. 1, 2016.

https://doi.org/10.1142/S0218396X15500162

[32] W.R. Watson, A method for determining acoustic-liner admittance in ducts with sheared flow in two-cross-sectional directions, 1985.

[33] N. Lamarque, T. Poinsot, Boundary Conditions for Acoustic Eigenmodes Computations in Gas Turbine Combustion Chambers, Aiaa Journal, Vol. 46, No. 9, pp. 2282-2292, 2015. https://doi.org/10.2514/1.35388

[34] S.K. Kim, H.S. Choi, H.J. Kim et al, Finite element analysis for acoustic characteristics of combustion stabilization devices, Aerospace Science and Technology, Vol. 42, pp. 229240, 2015. https://doi.org/10.1016/i.ast.2015.01.024

[35] T. Matsumoto, C.J. Zheng, S. Harada et.al, Explicit Evaluation of Hypersingular Boundary Integral Equation for 3-D Helmholtz Equation Discretized with Constant Triangular Element, Journal of Computational Science and Technology, Vol. 4, No. 3, Article ID 194, 2010. https://doi.org/10.1299/jcst.4.194

[36] N. Nishimura, Fast multipole accelerated boundary integral equation methods, Applied Mechanics Reviews, Vol. 55, No. 4, pp. 299-324, 2002.

https://doi.org/10.1115/1.1482087 
[37] S. Marburg, R. Anderssohn, Fluid structure interaction and admittance boundary conditions: setup of an analytical example, Journal of Computational Acoustics, Vol. 19, No. 1, pp. 63-74, 2011. https://doi.org/10.1142/S0218396X11004274

[38] O. Schenk, K. Gärtner, Solving Unsymmetric Sparse Systems of Linear Equations with PARDISO, Future Gener. Comput. Syst, Vol. 20, No. 3, pp. 475-487, 2004.

https://doi.org/10.1016/j.future.2003.07.011

[39] C.W. NELL, L.E. GILROY, An improved BASIS model for the BeTSSi submarine, Canada, DRDC - ATLANTIC, 2003. 